\title{
A TOPOLOGICAL REEB-MILNOR-ROSEN THEOREM AND CHARACTERIZATIONS OF MANIFOLDS
}

\author{
BY LOUIS F. McAULEY ${ }^{1}$ \\ Communicated by Steve Armentrout, July 8, 1971
}

Introduction. The following theorem is due to Reeb [9] (1952) and Milnor [7] (1956). All manifolds are differentiable $\left(C^{\infty}\right)$.

THEOREM A. Suppose that $M$ is a compact manifold and that $f$ is a differentiable real valued function on $M$ with exactly two critical points each of which is nondegenerate. Then $M$ is homeomorphic to a sphere.

If each critical point is degenerate, then the conclusion that $M$ is homeomorphic to a sphere is true. This was proved by Milnor [8] (1959) and by Rosen [10] (1960).

With the aid of some techniques of Dyer and Hamstrom, recent results of Kirby, Edwards, and Cernavskii on spaces of homeomorphism on manifolds, and a selection theorem of Michael it is not difficult to establish the following topological version of Theorem A.

THEOREM B. Suppose that $M$ is a continuum (compact connected metric space) and that $f: M \Rightarrow I=[0,1]$ is a (continuous) mapping. Furthermore, $f^{-1}(0)=a($ point $), f^{-1}(1)=b$ (point), $f \mid(M-\{a, b\})$ is completely regular, and $f^{-1}(x)$ is homeomorphic to an $n$-sphere $S^{n}$ for each $x \in(0,1)$. Then $M$ is homeomorphic to $S^{n+1}$.

Outline of A PROOF. Let $X=M-\{a, b\}$. For each $p \in(0,1)$, let $G_{p}$ denote the space of all homeomorphisms of $S^{n}$ onto $G_{p}$ with the usual metric (equivalently, the compact open topology). See, for example, [5] for details. The collection $G$ of all $G_{p}$ has the properties (1) $G^{*}$ (the union of the elements of $G$ ) is a complete metric space, (2) each $G_{p} \in G$ is $\mathrm{LC}^{\circ}$ (in the homotopy sense) by [1], [3], and (3) $G$ is equi-LC $\mathrm{C}^{\circ}$. It follows from $[\mathbf{2}, \mathbf{4}$, Theorems 4 , 5 , and the remark p. 113] that $M-\{a, b\} \cong S^{n} \times(0,1)$. Consequently, $M \cong S^{n+1}$.

REMARK. The condition that $f^{-1}(p) \cong S^{n}$ in Theorem B is a natural requirement since it follows from the hypothesis of Theorem $\mathrm{A}$ that the inverse of each point under $f$ is a manifold, in particular, a sphere of some fixed dimension $n$.

Characterization of manifolds. Similar characterizations of manifolds are possible although proofs are somewhat complicated by the existence of more than two topological "critical" points and "levels." Rather general

AMS 1969 subject classifications. Primary 5428, 5478, 5460, 5560, 5731, 5701.

${ }^{1}$ Research supported in part by NSF Grant GP 19589. 
theorems will be published elsewhere with sufficient details. The following special case illustrates the techniques involved and a simple but crucial idea which allows us to prove analogues of Theorem B for manifolds.

TheOREM C. Suppose that $M$ is a continuum and that $f: M \Rightarrow[0,1]$ is a mapping such that (1) $f^{-1}(0)=a$ (point), (2) $f^{-1}(1)=b$ (point), (3) $f^{-1}\left(\frac{1}{4}\right)$ $\cong f^{-1}\left(\frac{3}{4}\right) \cong$ a figure eight (two circles with exactly one common point), (4) for $0<x<\frac{1}{4}$ or $\frac{3}{4}<x<1, f^{-1}(x) \cong a$ circle, (5) for $\frac{1}{4}<x<\frac{3}{4}$, $f^{-1}(x) \cong$ a pair of disjoint circles, and (6) for $0<x<1$, there is a "triangulation" of $f^{-1}(x)$ which contains exactly four 1-simplexes (simple arcs) and $f$ is completely regular with respect to the collection of all 1-simplexes (defined below). Then $M \cong$ Torus or a Klein bottle.

Before giving a sketch of a proof, we define what we mean by a completely regular mapping with respect to a collection of arcs as used above. That is, given $\varepsilon>0$, there is a $\delta>0$ so that if $p, q \in(0,1)$ and $|p-q|<\delta$, then the four 1-simplexes of $f^{-1}(p)$ can be mapped by homeomorphisms $h_{1}, h_{2}, h_{3}$, and $h_{4}$ onto the four 1-simplexes of $f^{-1}(q)$ so that no point of $f^{-1}(p)$ is moved more than $\varepsilon$. We say that $f^{-1}(p)$ is piecewise homeomorphic to $f^{-1}(q)$.

OUTLINE OF A PROOF OF THEOREM C. Let $C$ denote a circle triangulated into exactly four 1-simplexes (also four vertices). If $C_{1}$ and $C_{2}$ are a pair of disjoint circles triangulated into exactly four 1-simplexes, then the space of all quadruples of homeomorphisms (4-piece homeomorphisms) of $C$ onto $C_{1} \cup C_{2}$ taking the 1-simplexes of $C$ onto the 1-simplexes of $C_{1} \cup C_{2}$ is locally arcwise connected. Furthermore, this space is homeomorphic to the space of all 4-piece homeomorphisms of $C$ onto $C$ (fixed triangulation). For each $x \in(0,1)$ and fixed triangulation of $C$, let $G_{x}$ denote the space of all 4-piece homeomorphisms of $C$ onto $f^{-1}(x)$. Now, let $G$ denote the collection of all $G_{x}$ and $G^{*}$ denote the union of the elements of $G$. It follows that (1) $G^{*}$ is a complete metric space, (2) $G_{x}$ for each $x \in(0,1)$ is $\mathrm{LC}^{\circ}$, and (3) $G$ is equi-LC ${ }^{\circ}$

An application of Michael's selection theorem as given in the proof of Theorem 4 of [2], [4] yields the desired result.

Characterizations of $n$-manifolds may be obtained in a similar manner. Detailed proofs of the more general theorems will appear elsewhere.

\section{BIBLIOGRAPHY}

1. A. V. Černavskiǐ, Local contractibility of the homeomorphism group of a manifold, Dokl. Akad. Nauk SSSR 182 (1968), 510-513 = Soviet Math. Dokl. 9 (1968), 1171-1174. MR 38 \# 5241 .

2. E. Dyer and M.-E. Hamstrom, Completely regular mappings, Fund. Math. 45 (1958), 103-118. MR 19, 1187.

3. R. C. Edwards and R. C. Kirby, Deformations of spaces of imbeddings, Ann. of Math. 93 (1971), 63-88.

4. M.-E. Hamstrom, Completely regular mappings whose inverses have $\mathrm{LC}^{\circ}$ homeomorphism group: A correction, Proc. Conf. on Monotone Mappings and Open Mappings, SUNY, Binghampton, 1970, pp. 255-260. 
5. L. F. McAuley, The existence of a complete metric for a special mapping space and some consequences, Topology Seminar (Wisconsin, 1965), Ann. of Math. Studies, no. 60, Princeton Univ. Press, Princeton, N.J., 1966, pp. 135-139. MR 37 \# 6903.

6. E. A. Michael, Continuous selections. I, II, III, Ann. of Math. (2) 63 (1956), 361-382; ibid. (2) 64 (1956), 562-580; ibid. (2) 65 (1957), 375-390. MR 17, 990; 18, 325; 750

7. J. W. Milnor, On manifolds homeomorphic to the 7-sphere, Ann. of Math. (2) 64 (1956), 399-405. MR 18, 498.

8. Soc. Math. France 87 (1959), 439-444. MR 22 \#8518.

9. G. Reeb, Sur certaines propriétés topologiques des variétés feuilletées, Actualités Sci. Indust., no. 1183 = Publ. Inst. Math. Univ. Strasbourg, Hermann, Paris, 1952, pp. 91-154, 157-158. MR 14, 1113.

10. R. Rosen, $A$ weak form of the star conjecture for manifolds, Notices Amer. Math. Soc. 7 (1960), 380. Abstract \# 570-28.

Department of Mathematics, State University of New York at Binghamton, Binghamton, NeW YORK 13901 